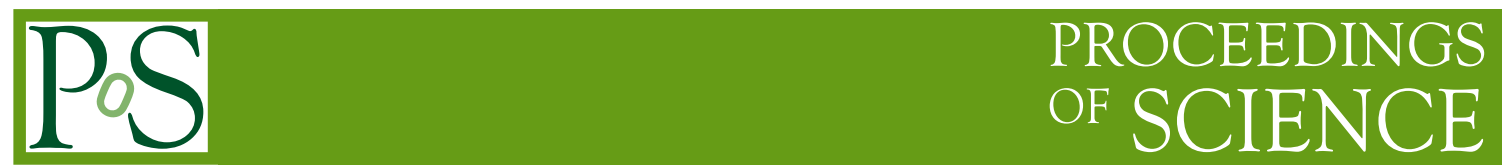

\title{
M-theory and matrix models
}

\author{
Sanefumi Moriyama* \\ Kobayashi-Maskawa Institute, Nagoya University \\ Graduate School of Mathematics, Nagoya University \\ E-mail: moriyama@math.nagoya-u.ac.jp
}

\begin{abstract}
We summarize our results on the $\mathrm{ABJ}(\mathrm{M})$ matrix model, which is the vacuum expectation value of a supersymmetric Wilson loop operator in the M2-brane worldvolume theory. We first give a Giambelli formula for the $\mathrm{ABJ}(\mathrm{M})$ matrix model. This formula implies that the fractional brane can be interpreted as a composite state of string excitations. We also present an exact instanton expansion of the ABJM partition function. This instanton expansion is especially interesting, because the coefficients of the string worldsheet instanton are divergent at certain Chern-Simons levels, though the divergences are completely cancelled by the divergences coming from the coefficients of the other membrane instanton. This is reminiscent of the lessons we learned in the non-perturbative study of string theory: String theory is a consistent theory only after including the non-perturbative branes.
\end{abstract}

KMI International Symposium 2013 on "Quest for the Origin of Particles and the Universe", 11-13 December, 2013

Nagoya University, Japan

\footnotetext{
*Speaker.
} 


\section{Introduction}

In physics, it is standard to study the most special background first, where sometimes symmetry is enhanced and sometimes the model becomes simple or even solvable, and then proceed to more general backgrounds by perturbation theory or other methods. In string theory, in some sense, the five perturbative string theories on ten-dimensional flat spacetime served the role of the special backgrounds. However, when the non-perturbative effects in string theory became clearer about two decades ago, the situation changed drastically. We found that, in the strong coupling limit, both the type IIA string theory and the heterotic $E_{8} \times E_{8}$ string theory become a theory with an enhanced Lorentz symmetry dubbed M-theory, whose low energy effective action is the eleven-dimensional supergravity. Hence, the most special background would be M-theory from this non-perturbative viewpoint.

Since the number of the supercharges is also maximal if we take the superconformal symmetry into account, it is equivalently fundamental to study the backgrounds, type IIB string theory on $A d S_{5} \times S^{5}$, M-theory on $A d S_{4} \times S^{7}$ and M-theory on $A d S_{7} \times S^{4}$, whose gauge theory duals are D3-branes, M2-branes and M5-branes respectively. Even though these theories share the special property of the maximal supersymmetry, they are still some of the most mysterious theories. Therefore, we are far from satisfied with our understanding of M-theory or non-perturbative string theory. One strategy to attack this situation would be to clarify the mathematical structures of these special backgrounds first and try to read off as much physical implication as possible.

Here let us concentrate on the M2-brane case. We shall obtain some physical insights by studying the worldvolume theory deeply. It was proposed in [1, 2] that the worldvolume theory of $\min \left(N_{1}, N_{2}\right)$ M2-branes with $\left|N_{2}-N_{1}\right|$ fractional M2-branes on the geometry $\mathbb{C}^{4} / \mathbb{Z}_{k}$ is described by $\mathscr{N}=6$ supersymmetric Chern-Simons theory with gauge group $U\left(N_{1}\right) \times U\left(N_{2}\right)$ and levels $k,-k$. It was also found that, due to the localization techniques [3], partition function and vacuum expectation values of BPS Wilson loops, originally defined by an infinite-dimensional path integral, are reduced to a finite-dimensional matrix integration [4, 5, 6]. This matrix model is called the $\mathrm{ABJ}(\mathrm{M})$ matrix model. In the following sections, we shall first present our results on this matrix model in a rather mathematical language, so that the statements look clearer, and then proceed to discuss their physical implication.

\section{Half-BPS Wilson loop in arbitrary representations}

Let us first summarizes our result of [7, 8]. We define the $\mathrm{ABJ}(\mathrm{M})$ matrix model in canonical ensemble as

$$
\begin{aligned}
\left\langle s_{\lambda}\right\rangle_{k}\left(N_{1}, N_{2}\right)= & \frac{(-1)^{\frac{1}{2} N_{1}\left(N_{1}-1\right)+\frac{1}{2} N_{2}\left(N_{2}-1\right)}}{N_{1} ! N_{2} !} \int \frac{d^{N_{1}} \mu}{(2 \pi)^{N_{1}}} \frac{d^{N_{2}} v}{(2 \pi)^{N_{2}}} s_{\lambda}\left(e^{\mu_{1}}, \ldots, e^{\mu_{N_{1}}} \mid e^{v_{1}}, \ldots, e^{v_{N_{2}}}\right) \\
& \times\left(\frac{\prod_{i<j} 2 \sinh \frac{\mu_{i}-\mu_{j}}{2} \prod_{a<b} 2 \sinh \frac{v_{a}-v_{b}}{2}}{\prod_{i, a} 2 \cosh \frac{\mu_{i}-v_{a}}{2}}\right)^{2} e^{\frac{i k}{4 \pi}\left(\sum_{i} \mu_{i}^{2}-\sum_{a} v_{a}^{2}\right)}
\end{aligned}
$$

This matrix model was obtained by computing the partition function or the vacuum expectation value of a half-BPS Wilson loop in the ABJ(M) theory with the localization techniques [4, 9, 10]. 
To reduce the explanation, here let us simply accept it as a definition and explain its structure in the following.

Let us first understand this matrix model from the group-theoretical viewpoint. The most fundamental matrix model is probably the Gaussian matrix model

$$
\frac{1}{N !} \int \frac{d^{N} \mu}{(2 \pi)^{N}} s_{\lambda}\left(e^{\mu_{1}}, \ldots, e^{\mu_{N_{1}}}\right)\left(\prod_{i<j}\left(\mu_{i}-\mu_{j}\right)\right)^{2} e^{-\frac{k}{4 \pi} \sum_{i} \mu_{i}^{2}} .
$$

There are two natural ways to deform this model. One deformation is the supersymmetrization: We can replace the $U(N)$ invariant measure by the invariant measure of the supergroup $U\left(N_{1} \mid N_{2}\right)$. Another is the Chern-Simons deformation or the so-called $q$-deformation. We replace the integration variable $\mu$ by its exponential function $e^{\mu}$. The $\mathrm{ABJ}(\mathrm{M})$ matrix model can be regarded as the Gaussian matrix model with the two simultaneous deformations. Therefore, it is suitable to call the $\mathrm{ABJ}(\mathrm{M})$ matrix model the supersymmetric Chern-Simons matrix model as well.

Here $s_{\lambda}\left(e^{\mu_{1}}, \ldots, e^{\mu_{N_{1}}} \mid e^{v_{1}}, \ldots, e^{V_{N_{2}}}\right)$ is the supersymmetric Schur polynomial depending on the partition $\lambda$. The supersymmetric Schur polynomial is the character of the supergroup $U\left(N_{1} \mid N_{2}\right)$ in a representation $\lambda$.

As a special case, when the representation is trivial, $s_{\lambda=.}=1$, the matrix integration corresponds to the partition function, while for a non-trivial representation, the matrix integration corresponds to the vacuum expectation value of a half-BPS Wilson loop. When $N_{2}=N_{1}$, let us call the $\mathrm{ABJ}(\mathrm{M})$ matrix model the ABJM matrix model, while when $N_{2} \neq N_{1}$ we call it the ABJ matrix model. In this sense, the ABJM partition function with $N_{2}=N_{1}$ and $s_{\lambda=.}=1$ is the most fundamental matrix integration here.

Hereafter, without loss of generality, we assume $M=N_{2}-N_{1} \geq 0$ and $k>0$.

\subsection{Generalized Giambelli compatibility}

Let us also define the $\mathrm{ABJ}(\mathrm{M})$ matrix model in grand canonical ensemble as

$$
\left\langle s_{\lambda}\right\rangle_{k, M}^{\mathrm{GC}}(z)=\sum_{N=0}^{\infty} z^{N}\left\langle s_{\lambda}\right\rangle_{k}(N, N+M)
$$

and define the one normalized by the ABJM partition function with a double bracket,

$$
\left\langle\left\langle s_{\lambda}\right\rangle\right\rangle_{k, M}^{\mathrm{GC}}(z)=\frac{\left\langle s_{\lambda}\right\rangle_{k, M}^{\mathrm{GC}}(z)}{\langle 1\rangle_{k, 0}^{\mathrm{GC}}(z)}
$$

Then, we find a theorem [7, 8, 11] stating that the normalized grand canonical vacuum expectation value can be expressed by a determinant

$$
\left.\left\langle s_{\lambda}\right\rangle\right\rangle_{k, M}^{\mathrm{GC}}(z)=\operatorname{det}\left(\left(H_{l_{p},-M+q-1}(z)\right)_{\substack{1 \leq p \leq M+r \\ 1 \leq q \leq M}} \mid\left(\widetilde{H}_{l_{p}, a_{q}}(z)\right)_{\substack{1 \leq p \leq M+r \\ 1 \leq q \leq r}}\right),
$$

where $H_{p, q}(z)$ and $\widetilde{H}_{p, q}(z)$ are given by

$$
H_{p, q}(z)=E_{p} \bullet[1+z \boldsymbol{Q} \circ \boldsymbol{P} \bullet]^{-1} E_{q}, \quad \widetilde{H}_{p, q}(z)=z E_{p} \bullet[1+z \boldsymbol{Q} \circ \boldsymbol{P} \bullet]^{-1} \boldsymbol{Q} \circ E_{q} .
$$




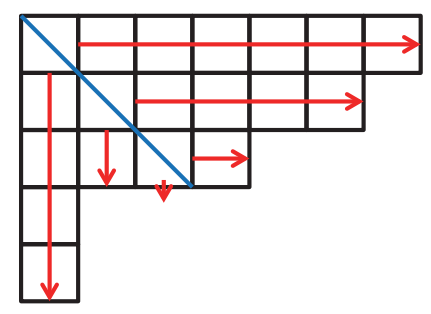

(a)

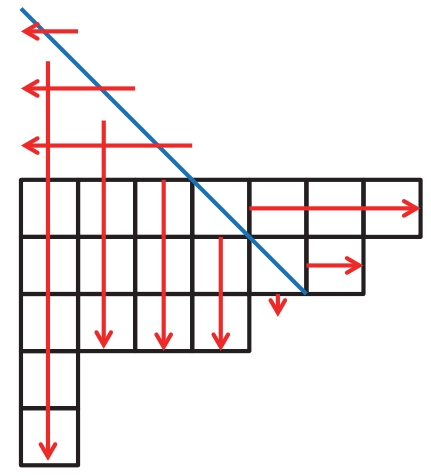

(b)

Figure 1: Frobenius notation for the ABJM case (a) and for the ABJ case (b). The same Young diagram is expressed as $\left(a_{1} a_{2} a_{3} \mid l_{1} l_{2} l_{3}\right)=(641 \mid 410)$ in the ABJM case while $\left(a_{1} a_{2} \mid l_{1} l_{2} l_{3} l_{4} l_{5}\right)=(31 \mid 74320)$ in the ABJ case $(M=3)$. It is also convenient to regard the first three inverse horizontal arrows in (b) as additional arm lengths $(-1,-2,-3)$.

In the above expression, $\boldsymbol{P}, \boldsymbol{Q}$ and $E_{j}$ are regarded as "matrices or vectors" with continuous indices $\mu, v$, whose components are given by

$$
(\boldsymbol{P})_{\mu, v}=\frac{1}{2 \cosh \frac{\mu-v}{2}}, \quad(\boldsymbol{Q})_{v, \mu}=\frac{1}{2 \cosh \frac{v-\mu}{2}}, \quad\left(E_{j}\right)_{v}=e^{\left(j+\frac{1}{2}\right) v} .
$$

In (2.6) the matrix multiplications $\circ$ and $\bullet$ are defined by "contracting" these continuous indices $\mu$, $v$, in the sense of integration with the following measures

$$
\int \frac{d \mu}{2 \pi} e^{\frac{i k}{4 \pi} \mu^{2}}, \quad \int \frac{d v}{2 \pi} e^{-\frac{i k}{4 \pi} v^{2}}
$$

In (2.5) the non-negative integers $a_{q}$ and $l_{p}$ are the numbers appearing in the modified Frobenius symbol $\left(a_{1} \cdots a_{r} \mid l_{1} \cdots l_{r+M}\right)$, which is defined by

$$
a_{q}=\lambda_{q}-q-M, \quad l_{p}=\lambda_{p}^{\prime}-p+M,
$$

with

$$
r=\max \left\{s \mid \lambda_{s}-s-M \geq 0\right\}=\max \left\{s \mid \lambda_{s}^{\prime}-s+M \geq 0\right\}-M .
$$

Pictorially these are the numbers of boxes counted from the diagonal line shifted by $M$. (See figure 1.)

As a corollary [7], for the special case of the ABJM matrix model $M=0$, we find the relation

$$
\left\langle\left\langle s_{\left(a_{1}, \cdots, a_{r} \mid l_{1}, \cdots, l_{r}\right)}\right\rangle\right\rangle_{k, 0}^{\mathrm{GC}}(z)=\operatorname{det}\left(\left\langle\left\langle s_{\left(a_{q} \mid l_{p}\right)}\right\rangle\right\rangle_{k, 0}^{\mathrm{GC}}(z)\right)_{\substack{1 \leq p \leq r \\ 1 \leq q \leq r}} .
$$

It is a classical mathematical result stating that the Schur polynomial itself (and its supersymmetric generalization) satisfies the Giambelli formula

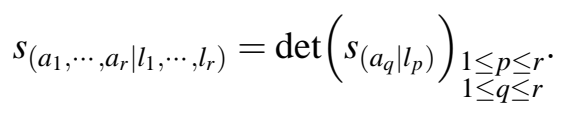




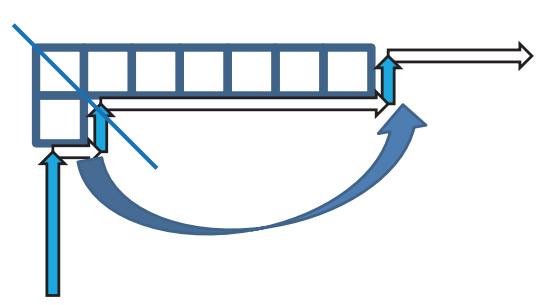

(a)

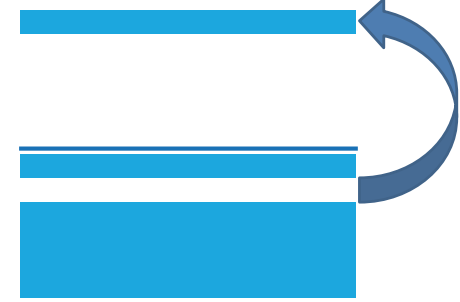

(b)

Figure 2: The hook representation $(a \mid l)$ can be regarded as the fundamental excitation of a fermion, where the fermion in the $(l+1)$-st state below the sea level of the Dirac sea is excited to the $(a+1)$-st state above the sea level.

Hence, our relation (2.11) claims that we can put the normalized grand canonical expectation values $\left\langle\langle\cdot\rangle_{k, 0}^{\mathrm{GC}}\right.$ to all of the characters appearing in the Giambelli formula (2.12). In other words, the formula (2.11) can be stated that the normalized grand canonical expectation values of the halfBPS Wilson loop in the ABJM matrix model is Giambelli compatible and the original formula (2.5) shows the Giambelli compatibility in a more general sense.

Notice that, even for the $\mathrm{ABJ}$ partition function $\left(N_{2} \neq N_{1}\right)$ where there is no Young diagram, the effect of shifting the diagonal line in the Frobenius symbol still causes a non-trivial determinant,

$$
\left.\frac{\langle 1\rangle_{k, M}^{\mathrm{GC}}(z)}{\langle 1\rangle_{k, 0}^{\mathrm{GC}}(z)}=\operatorname{det}\left(\left(H_{M-p,-M+q-1}(z)\right)\right)_{\substack{1 \leq p \leq M \\ 1 \leq q \leq M}}\right) .
$$

\subsection{ABJ fractional branes from ABJM Wilson loop}

Now let us present a physical interpretation for the generalized Giambelli formula (2.5), (2.13).

The Young diagram can be regarded as the fermion excitation. Namely, we place the Young diagram on the upper left corner and trace its outline. We identify the vertical line as the states filled by the fermions and the horizontal line as the states unfilled. With this rule, the hook representation is identified as a fundamental excitation of a single fermion, while a non-hook one is as a collective excitation of multiple fermions. (See figure 2.) Here we also see that the diagonal line is interpreted as the sea level of the Dirac sea. Hence, the shift of the diagonal line in the Frobenius symbol in the ABJ matrix model $\left(N_{2} \neq N_{1}\right)$ can be interpreted as a highly-complicated solitonic excitation of fermions.

Since the Wilson loop in the fundamental representation has the same charge as a string, this fermion can be interpreted as a string excitation. Compared with the ABJM theory, the new physical object appearing in the ABJ theory is the fractional brane. Hence, the formula (2.13) states that the fractional brane can be considered as a highly-complicated solitonic excitation of strings.

This is similar to the excitations considered in the AdS/CFT correspondence [12]. As the gauge theory operator becomes bigger and bigger, small fluctuating gravitational mode becomes a heavier brane or even changes the background geometry. 


\section{Instanton effects}

So far we have considered a rather kinematical structure of the $\mathrm{ABJ}(\mathrm{M})$ matrix model. Let us turn to a more dynamical instanton effect next.

\subsection{Exact instanton expansions}

For simplicity let us consider the ABJM partition function. From a series of works [13, 14, 15, 16, 11, 17, 18, 19, 20, 21, 22], we have found the grand potential of the ABJM partition function defined by

$$
e^{J_{k}(\mu)}=\langle 1\rangle_{k, 0}^{\mathrm{GC}}\left(e^{\mu}\right)
$$

has the expansion

$$
J_{k}(\mu)=J_{k}^{\mathrm{pert}}\left(\mu^{\mathrm{eff}}\right)+J_{k}^{\mathrm{np}}\left(\mu^{\mathrm{eff}}\right) .
$$

The perturbative part is given by [15, 16, 11]

$$
J_{k}^{\text {pert }}(\mu)=\frac{C}{3} \mu^{3}+B \mu+A,
$$

with the perturbative coefficients being

$$
\begin{aligned}
& C=\frac{2}{\pi^{2} k}, \quad B=\frac{1}{3 k}+\frac{k}{24}, \\
& A=-\frac{1}{6} \log \frac{k}{4 \pi}+2 \zeta^{\prime}(-1)-\frac{\zeta(3)}{8 \pi^{2}} k^{2}+\frac{1}{3} \int \frac{d x}{e^{k x}-1}\left(\frac{3}{x \sinh ^{2} x}-\frac{3}{x^{3}}+\frac{1}{x}\right),
\end{aligned}
$$

while the non-perturbative part is given by $[19,20,21,22]\left(\lambda_{s}=2 / k\right)$

$$
J_{k}^{(n p)}\left(\mu^{\mathrm{eff}}\right)=F_{\mathrm{top}}\left(T_{1}^{\mathrm{eff}}, T_{2}^{\mathrm{eff}}, \lambda_{s}\right)+\frac{1}{2 \pi i} \frac{\partial}{\partial \lambda_{s}}\left[\lambda_{s} F_{\mathrm{NS}}\left(\frac{T_{1}^{\mathrm{eff}}}{\lambda_{s}}, \frac{T_{2}^{\mathrm{eff}}}{\lambda_{s}}, \frac{1}{\lambda_{s}}\right)\right],
$$

where $F_{\text {top }}(\boldsymbol{T}, \tau)$ and $F_{\mathrm{NS}}(\boldsymbol{T}, \tau)$ are respectively defined by the free energy of the refined topological string theory

$$
\begin{aligned}
& F_{\mathrm{ref}}\left(\boldsymbol{T}, \tau_{1}, \tau_{2}\right)=\sum_{j_{L}, j_{R} \geq 0} \sum_{\boldsymbol{d}} \sum_{n=1}^{\infty} \frac{N_{j_{L}, j_{R}}^{\boldsymbol{d}} \chi_{j_{L}}\left(q_{L}^{n}\right) \chi_{j_{R}}\left(q_{R}^{n}\right)}{n\left(q_{1}^{n / 2}-q_{1}^{-n / 2}\right)\left(q_{2}^{n / 2}-q_{2}^{-n / 2}\right)} e^{-n \boldsymbol{d} \cdot \boldsymbol{T}}, \\
& \chi_{j}(q)=\frac{q^{2 j+1}-q^{-2 j-1}}{q-q^{-1}}, \quad q_{L, R}=e^{\pi i\left(\tau_{1} \mp \tau_{2}\right)}, \quad q_{1,2}=e^{2 \pi i \tau_{1,2}}
\end{aligned}
$$

in the topological limit and in the Nekrasov-Shatashvilli limit,

$$
F_{\text {top }}(\boldsymbol{T}, \tau)=\lim _{\substack{\tau_{1} \rightarrow \tau \\ \tau_{2} \rightarrow-\tau}} F_{\text {ref }}\left(\boldsymbol{T}, \tau_{1}, \tau_{2}\right), \quad F_{\mathrm{NS}}(\boldsymbol{T}, \tau)=\lim _{\substack{\tau_{1} \rightarrow \tau \\ \tau_{2} \rightarrow 0}} 2 \pi i \tau_{2} F_{\text {ref }}\left(\boldsymbol{T}, \tau_{1}, \tau_{2}\right) .
$$

Here $T_{1,2}^{\text {eff }}$ are two Kahler parameters identified with the chemical potential

$$
T_{1,2}^{\mathrm{eff}}=\frac{4 \mu^{\mathrm{eff}}}{k} \pm \pi i,
$$

modified by [21]

$$
\mu^{\mathrm{eff}}=\left\{\begin{array}{l}
\mu-(-1)^{k / 2} 2 e^{-2 \mu}{ }_{4} F_{3}\left(1,1, \frac{3}{2}, \frac{3}{2} ; 2,2,2 ;(-1)^{k / 2} 16 e^{-2 \mu}\right), \\
\mu+e^{-4 \mu_{4} F_{3}}\left(1,1, \frac{3}{2}, \frac{3}{2} ; 2,2,2 ;-16 e^{-4 \mu}\right) .
\end{array}\right.
$$




\subsection{Cancellation mechanism}

In the above subsection, we have presented an exact instanton expansion of the ABJM grand potential. After including all the instanton effects, we finally arrive at the very compact expression (3.3), (3.5). However, to distinguish each instanton contribution, let us expand it slightly

$$
J_{k}(\mu)=\frac{C}{3} \mu^{3}+B \mu+A+\sum_{\substack{\ell, m=0 \\(\ell, m) \neq(0,0)}}^{\infty} f_{\ell, m}(\mu) e^{-2 \ell \mu-\frac{4 m \mu}{k}},
$$

where $f_{\ell, m}(\mu)$ is a quadratic function of $\mu$ for $m=0$ and a constant function for other cases. We call the terms with $\ell=0$ as worldsheet instanton terms, those with $m=0$ as membrane instanton terms and those with $m \neq 0, \ell \neq 0$ as bound state terms, since the $\ell=0$ terms are identified as the string worldsheet wrapping the holomorphic cycle $\mathbb{C P}^{1} \subset \mathbb{C P}^{3}[23,13]$ while the $m=0$ terms are identified with D2-branes wrapping the Lagrangian submanifold $\mathbb{R P}^{3} \subset \mathbb{C P}^{3}$ [14].

From explicit numerical studies, we find that the coefficient is vanishing when both the level $k$ and the membrane instanton number $\ell$ are odd integers. So for integers $k$, we obtain a schematic expansion with all the finite coefficients omitted as in figure 3. We first observe that when the instanton number is smaller than $k / 2$, the coefficients match correctly with those of the worldsheet instanton predicted from the topological string theory. We further find that the coefficients of the worldsheet instanton are divergent at certain levels. If we require that these divergences are cancelled by the divergences of the coefficients in the membrane instanton, we can determine the coefficients in the membrane instanton for general $k[19,20]$, so that they also match with the WKB analysis in [11].

This cancellation mechanism meets our expectation in string democracy. Namely, only the string worldsheet instanton will cause a divergent theory. It is only after we include the membrane instanton coming from the non-perturbative branes that the divergences cancel among them.

Note that the cancellation mechanism is not only important from the above aesthetic viewpoint, but also for the practical reason. In fact, let us stress that the first few membrane instantons are determined from this cancellation mechanism in [19, 20] and our exact instanton expansion (3.5) is confirmed by this cancellation mechanism [22].

The analysis for the ABJM partition function was also extended to the case of the half-BPS Wilson loop expectation values in [7] and the case of the ABJ partition function in [8].

\section{Acknowledgements}

I am grateful to all of my collaborators for many valuable discussions. I would also like to thank H. Itoyama, H. Kanno and M. Marino for their constant supports on this project. I am also grateful to the KMI visitor program, which enables the intensive lecture by M. Marino at Kobayashi-Maskawa Institute in 2013 and the collaboration [22].

\section{References}

[1] O. Aharony, O. Bergman, D. L. Jafferis and J. Maldacena, "N=6 superconformal Chern-Simons-matter theories, M2-branes and their gravity duals," JHEP 0810, 091 (2008) [arXiv:0806.1218 [hep-th]]. 


$$
\begin{aligned}
& J_{k=1}(\mu)=e^{-4 \mu}+e^{-8 \mu}+e^{-12 \mu}+e^{-16 \mu}+e^{-20 \mu}+e^{-24 \mu}+\cdots \\
& J_{k=2}(\mu)=e^{-2 \mu}+e^{-4 \mu}+e^{-6 \mu}+e^{-8 \mu}+e^{-10 \mu}+e^{-12 \mu}+\cdots \\
& J_{k=3}(\mu)=e^{-\frac{4}{3} \mu}+e^{-\frac{8}{3} \mu}+e^{-4 \mu}+e^{-\frac{16}{3} \mu}+e^{-\frac{20}{3} \mu}+e^{-8 \mu}+\cdots
\end{aligned}
$$

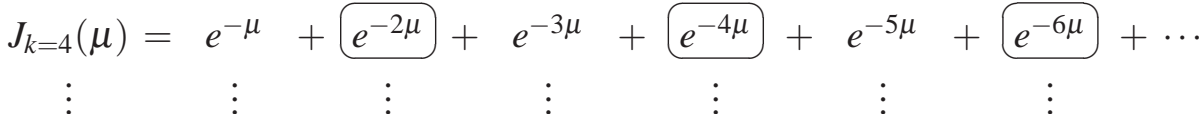

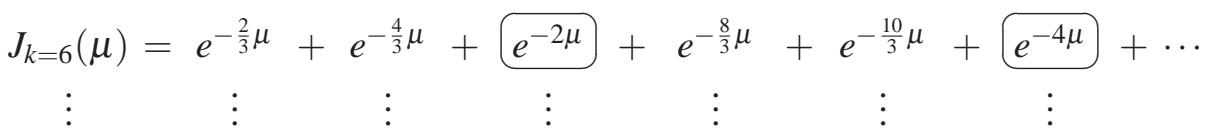

Figure 3: The schematic instanton expansion for various integers $k$ with their coefficients omitted. We encircle terms where the coefficients of the worldsheet instanton are divergent. The divergences are cancelled by the coefficients of the membrane instanton.

[2] O. Aharony, O. Bergman and D. L. Jafferis, "Fractional M2-branes,” JHEP 0811, 043 (2008) [arXiv:0807.4924 [hep-th]].

[3] V. Pestun, "Localization of gauge theory on a four-sphere and supersymmetric Wilson loops," Commun. Math. Phys. 313, 71 (2012) [arXiv:0712.2824 [hep-th]].

[4] A. Kapustin, B. Willett and I. Yaakov, "Exact Results for Wilson Loops in Superconformal Chern-Simons Theories with Matter," JHEP 1003, 089 (2010) [arXiv:0909.4559 [hep-th]].

[5] D. L. Jafferis, “The Exact Superconformal R-Symmetry Extremizes Z,” JHEP 1205, 159 (2012) [arXiv:1012.3210 [hep-th]].

[6] N. Hama, K. Hosomichi and S. Lee, "Notes on SUSY Gauge Theories on Three-Sphere," JHEP 1103, 127 (2011) [arXiv:1012.3512 [hep-th]].

[7] Y. Hatsuda, M. Honda, S. Moriyama and K. Okuyama, "ABJM Wilson Loops in Arbitrary Representations," JHEP 1310, 168 (2013) [arXiv:1306.4297 [hep-th]].

[8] S. Matsumoto and S. Moriyama, “ABJ Fractional Brane from ABJM Wilson Loop,” JHEP 1403, 079 (2014) [arXiv:1310.8051 [hep-th]].

[9] N. Drukker and D. Trancanelli, "A Supermatrix model for N=6 super Chern-Simons-matter theory," JHEP 1002, 058 (2010) [arXiv:0912.3006 [hep-th]].

[10] M. Marino and P. Putrov, "Exact Results in ABJM Theory from Topological Strings," JHEP 1006, 011 (2010) [arXiv:0912.3074 [hep-th]].

[11] M. Marino and P. Putrov, “ABJM theory as a Fermi gas,” J. Stat. Mech. 1203, P03001 (2012) [arXiv:1110.4066 [hep-th]].

[12] H. Lin, O. Lunin and J. M. Maldacena, "Bubbling AdS space and 1/2 BPS geometries," JHEP 0410, 025 (2004) [hep-th/0409174].

[13] N. Drukker, M. Marino and P. Putrov, "From weak to strong coupling in ABJM theory," Commun. Math. Phys. 306, 511 (2011) [arXiv:1007.3837 [hep-th]].

[14] N. Drukker, M. Marino and P. Putrov, “Nonperturbative aspects of ABJM theory," JHEP 1111, 141 (2011) [arXiv:1103.4844 [hep-th]]. 
[15] H. Fuji, S. Hirano and S. Moriyama, "Summing Up All Genus Free Energy of ABJM Matrix Model," JHEP 1108, 001 (2011) [arXiv:1106.4631 [hep-th]].

[16] M. Hanada, M. Honda, Y. Honma, J. Nishimura, S. Shiba and Y. Yoshida, "Numerical studies of the ABJM theory for arbitrary N at arbitrary coupling constant," JHEP 1205, 121 (2012) [arXiv:1202.5300 [hep-th]].

[17] Y. Hatsuda, S. Moriyama and K. Okuyama, "Exact Results on the ABJM Fermi Gas," JHEP 1210, 020 (2012) [arXiv:1207.4283 [hep-th]].

[18] P. Putrov and M. Yamazaki, "Exact ABJM Partition Function from TBA," Mod. Phys. Lett. A 27, 1250200 (2012) [arXiv:1207.5066 [hep-th]].

[19] Y. Hatsuda, S. Moriyama and K. Okuyama, "Instanton Effects in ABJM Theory from Fermi Gas Approach,” JHEP 1301, 158 (2013) [arXiv:1211.1251 [hep-th]].

[20] F. Calvo and M. Marino, “Membrane instantons from a semiclassical TBA,” JHEP 1305, 006 (2013) [arXiv:1212.5118 [hep-th]].

[21] Y. Hatsuda, S. Moriyama and K. Okuyama, "Instanton Bound States in ABJM Theory," JHEP 1305, 054 (2013) [arXiv:1301.5184 [hep-th]].

[22] Y. Hatsuda, M. Marino, S. Moriyama and K. Okuyama, "Non-perturbative effects and the refined topological string," arXiv:1306.1734 [hep-th].

[23] A. Cagnazzo, D. Sorokin and L. Wulff, “String instanton in AdS(4) x CP**3," JHEP 1005, 009 (2010) [arXiv:0911.5228 [hep-th]]. 